\title{
Erratum to: Normal karyotype acute myeloid leukemia patients with $C E B P A$ double mutation have a favorable prognosis but no survival benefit from allogeneic stem cell transplant
}

\author{
Jae-Sook Ahn ${ }^{1}$ • Jae-Yong Kim ${ }^{1}$ • Hyeoung-Joon Kim ${ }^{1}$ • Yeo-Kyeoung Kim ${ }^{1}$. \\ Seung-Shin Lee ${ }^{1} \cdot$ Sung-Hoon Jung ${ }^{1}$ • Deok-Hwan Yang ${ }^{1} \cdot$ Je-Jung Lee ${ }^{1}$ • \\ Nan Young Kim ${ }^{2} \cdot$ Seung Hyun Choi ${ }^{2}$ - Mark D. Minden ${ }^{3}$. Chul Won Jung ${ }^{4}$. \\ Jun-Ho Jang ${ }^{4}$ • Hee Je Kim ${ }^{5}$. Joon Ho Moon ${ }^{6}$ Sang Kyun Sohn ${ }^{6}$ • Jong-Ho Won ${ }^{7}$ • \\ Sung-Hyun Kim ${ }^{8}$. Dennis Dong Hwan Kim ${ }^{3}$
}

Published online: 2 December 2015

(C) Springer-Verlag Berlin Heidelberg 2015

Erratum to: Annals of Hematology

DOI 10.1007/s00277-015-2540-7

The authors inadvertently wrote incorrectly the author's name: Jae-Young Kim should be corrected as Jae-Yong Kim. His affiliation is the same. He has no competing interests to declare.

The online version of the original article can be found at http://dx.doi.org/ 10.1007/s00277-015-2540-7.

Hyeoung-Joon Kim

hjoonk@chonnam.ac.kr

1 Department of Hematology-Oncology, Chonnam National, University Hwasun Hospital, 322 Seoyangro,

Hwasun, Jeollanamdo 519-763, Republic of Korea

2 Genomic Research Center for Hematopoietic Diseases, Chonnam National University Hwasun Hospital, Hwasun, Jeollanam-do, Republic of Korea

3 Department of Medical Oncology and Hematology, Princess Margaret Cancer Centre, University of Toronto, Toronto, Canada
4 Division of Hematology-Oncology, Samsung Medical Center, Seoul, South Korea

5 Department of Hematology, Cancer Research Institute, Seoul St. Mary's Hospital, Colllege of Medicine, The Catholic University of Korea, Seoul, South Korea

6 Department of Hematology-Oncology, Kyungpook National University Hospital, Daegu, South Korea

7 Department of Hematology-Oncology, Soon ChunHyangUniversity Hospital, Seoul, South Korea

8 Department of Hematology-Oncology, Dong-A University College of Medicine, Busan, South Korea 